- myocardial oxygen consumption (State 4, State 3), Respiratory Control Ratio (RCR), and adenosine diphosphate - oxygen index $(\mathrm{ADP} / \mathrm{O})$ and

- oxidative stress biomarkers

METHODS/STUDY POPULATION: Animal treatments. In the course of twelve-week the male Chinchilla rabbits $(n=10$ /group) received once a day a single dose, in group: A - sunflower oil (control animals); B - atherogenic 2\% hypercholesterolemic diet; C - atherogenic diet and ramipril $(1 \mathrm{mg} / \mathrm{kg})$ and $\mathrm{D}$ - ramipril $(1 \mathrm{mg} / \mathrm{kg})$ only. Animals were terminated in accordance with the U.K. "Animals (Scientific Procedures) Act." Isolation of mitochondria Mitochondria from rabbit heart were isolated by tissue digestion (trypsin), fractionation and differential centrifugation. Mitochondrial respiratory functional measures (State 4 - Basal; State 3 - ADP-stimulated respiration, $R C R$ and $A D P / O)$ and biochemical markers of oxidative damage (the nitrite level) were measured polarographically (Clark electrode, YSI, USA) and spectrophotometrically, respectively, in isolated heart mitochondrial suspensions. Statistics - All results are reported as means $\pm S D$. Comparisons between ramipril treated and control animals were performed by unpaired t-test or one-way ANOVA with a Tukey adjustment for multiple comparisons. A $P$ value $<0.05$ was considered significant for all tests. RESULTS/ANTICIPATED RESULTS: Plasma cholesterol levels: After a period of 12 weeks

- Plasma cholesterol levels in control rabbits (A) were low (1.36 \pm $0.23 \mathrm{mmol} / \mathrm{l})$.

- Cholesterol-fed rabbits became hypercholesterolemic and their plasma total cholesterol level was higher even than $10 \mathrm{mmol} / \mathrm{l}$. The level of total cholesterol in the high-cholesterol-diet group was significantly increased compared with the level in the normal-diet group $(\mathrm{p}<0.01)$.

- In the high-cholesterol-diet group treated with ramipril (C), the plasma cholesterol level was not affected by the drug ramipril $(10.54 \pm 1.31 \mathrm{mmol} / \mathrm{l})$. ACE-I ramipril did not infuence the concentration of total cholesterol.

- Plasma cholesterol levels in group D were low (1.46 \pm 0.29 $\mathrm{mmol} / \mathrm{l})$.

Mitochondria protein concentration: 10-15 mg per heart ( $0.5 \mathrm{mg} / \mathrm{ml}$ ). Mitochondrial actions of ACE-I ramipril: a typical mitochondrial oxygen consumption rates in State 4 (basal) and State $3(+\mathrm{ADP})$ in malate-energized heart mitochondria after 12 -week treatment protocol highlights that, comparing to the control group:

- Atherogenic $2 \%$ cholesterol diet (B) caused a decline in mitochondrial function (in both, State 3 and 4) (-25\%).

- Mitochondria from group C animals (treatment with ramipril along with $2 \%$ cholesterol diet) exhibited higher State 3 respiratory rates compared with group $B$.

- Mild inhibition of mitochondrial respiration was recorded in group $\mathrm{D}$, in both respiratory states (V4\&V3).

No significant difference between the groups were found regarding RCR. However, high significant statistical decrease $(\mathrm{p}<0.01)$ was found in group B regarding ADP/O ratio. Nitrite levels: Rabbits receiving a supplementation of $2 \%$ cholesterol for 12 weeks (B) showed an increase in nitrite levels $(115 \pm 21 \mathrm{nmol} / \mathrm{l}$ vs $50 \pm 9$ $\mathrm{nmol} / \mathrm{l}$ controls). However, It was attenuated by ramipril (C) $(85 \pm 16 \mathrm{nmol} / \mathrm{l})$. Strong correlations were found between State
3 respiratory rate and the nitrite level $(r=-.967, p<0.05)$. It means: the higher level of nitrite - the lower oxygen consumption rate. DISCUSSION/SIGNIFICANCE OF IMPACT: The beneficial effect of ramipril was not noticed on plasma cholesterol levels. However, ramipril improves rabbit heart mitochondrial bioenergetics function during the course of experimental hypercholesterolemia.

- In cholestrerol-supplemented hearts myocardial oxygen consumption was markedly reduced (State 4 and State 3) compared to controls. Administration of high-cholesterol diet decreased not only the respiratory activity of rabbit heart mitochondria (RHM), but also the sensitivity of respiratory chain to ADP (ADP/O), while concomitantly caused an increase in nitrite production. Possible explanation: high-fat diet affects the fluidity of mitochondrial membrane - Electron transport chain (ETC) may be damaged, and unable to support high rates of respiration (e.g. substantial cytochrome c could be lost).

- Administration of ACE-I ramipril along with cholesterol diet partially abolish reduction in MQO2 and improved coupling efficiency (ADP/O). Possible explanation: reduced coupling efficiency means the coupling mechanism itself is altered (e.g. the respiratory complexes slip and pump fewer protons than normal and less ATP is produced per oxygen consumed. Ramipril partially improved coupling efficiency and increased the amount of ATP per oxygen consumed.

- RCR - No significant difference between the groups were found. High RCR indicates good function (a high capacity for substrate oxidation and ATP turnover). Low RCR usually indicates dysfunction. However, there is no absolute RCR value that is diagnostic of dysfunctional mitochondria, because values are substrate- and tissue-dependent.

- NO• exerts metabolic control over mitochondrial respiration. Group B: The lowered state 3-respiration in heart mitochondria seems to contribute to the increased NO production, and elevated nitrite level.

- In a system as complex as OXPHOS, conclusions about overall efficiency must involve measurements of: mito membrane potential, proton transport, ATP synthesis and modular kinetic analisys.

Measurements both, respiration \& potentials will enable us to identify the primary site of effectors, allowing deeper quantitative analyses and better patients therapy.

4371

\section{The Role of B Cells in Keloid Formation}

Jaclyn B Anderson ${ }^{1}$, Alexander B Harrant, Nalu Navarro-Alvarez, Zhaohui Wang, Adrie van Bokhoven, Whitney High, Tae W Chong, and Christene A. Huang

${ }^{1}$ University of Colorado at Denver

OBJECTIVES/GOALS: Recent studies indicate B cells are involved in dermal fibroblast activation and collagen deposition in the skin. However, B cell distribution in epidermal and dermal layers is unknown. Here, We aim to characterize the distribution of B cells residing in normal skin and keloidal scars. METHODS/STUDY POPULATION: One abdominal normal skin sample and two keloid samples (ear and shoulder) were obtained from the University of 
Colorado Biorepository Core Facility and from the Plastic Surgery Clinics. Five micron sections from formalin-fixed paraffin-embedded samples were prepared for multiplex fluorescence immunohistochemistry by the Human Immunology \& Immunotherapy Initiative. We stained for CD20+, CD19+, and DAPI. Slides were imaged using Vectra $^{\circledR} 3$ scanning system from PerkinElmer. Images were analyzed in InForm ${ }^{\circledR}$ Tissue Finder, phenotpr, phenoptrReports by Akoya biosciences. RESULTS/ANTICIPATED RESULTS: We found a significant increase in the percentage of CD20 + and CD19+ B cells in keloid skin compared to normal skin tissue $(14.50 \%$ and $14.20 \%$ vs $6.47 \%$ and $7.56 \%$ of the total cells), respectively. Interestingly, we found that in the epidermis of keloid skin CD20+ cell were more abundant (14.46\%) whereas in the epidermis normal skin CD20+ cells were less predominant (5.14\%). In the dermis of keloid skin, CD20+ and $\mathrm{CD} 19+$ were in equal proportions $(13 \%)$ whereas in normal skin CD19+ cells were more predominant $(10.44 \%)$ compared to CD20+ cells (7.04\%). Dual positive B cells, CD19+/CD20+ cells, were more abundant in keloid dermis (11.06\%) compared to normal skin dermis (1.24\%). DISCUSSION/SIGNIFICANCE OF IMPACT: B cells are involved in fibroblast activation in diseases such as scleroderma and rheumatoid arthritis. With the increase of CD19+/CD20+ B cells in keloids, the role of $\mathrm{B}$ cells in keloid pathogenesis warrants further study. CD27 staining may determine if these are activated or follicular $B$ cells.

4353

\section{The Role of BCL2 Mediated Calcium Signaling on Leukemia Stem Cell Metabolism \\ Anagha Inguva ${ }^{1}$, Shanshan Pei ${ }^{2}$, Maria Amaya ${ }^{2}$, Brett Stevens ${ }^{2}$, Courtney Jones ${ }^{2}$, Daniel Pollyea ${ }^{2}$, and Craig Jordan ${ }^{2}$ \\ ${ }^{1}$ University of Colorado at Denver; ${ }^{2}$ University of Colorado}

OBJECTIVES/GOALS: The objective of this study is to define the molecular mechanisms that control survival of malignant stem cells in acute myeloid leukemia (AML). Leukemia stem cells (LSCs) are not effectively eradicated by standard treatment and lead to resistance and relapse, which contribute to poor survival rates. METHODS/STUDY POPULATION: The recently FDA approved venetoclax, a BCL2 inhibitor, with azacitidine, a hypomethylating agent leads to a $70 \%$ response rate in AML patients. Analysis of patients treated with this regimen showed direct targeting of LSCs. BCL2 has a non-canonical function in regulation of intracellular calcium. To determine how BCL2 mediated calcium signaling plays a role in LSC biology, we used LSCs isolated from venetoclax/ azacitidine (ven/aza) sensitive and resistant patient samples to measure expression of calcium channels via RNA seq. BIO-ID, siRNA, flow cytometry, seahorse assays, calcium measurements and colony assays were used to determine the effects of calcium channel perturbation on LSC biology. RESULTS/ANTICIPATED RESULTS: BCL2 inhibition leads to decreased OXPHOS activity in primary AML specimens. BIO-ID studies revealed cation/metal ion transporters, ER membrane proteins and ER membrane organization as top enriched pathways interacting with BCL2. RNA-seq data showed increased expression of genes involved in calcium influx into the ER in ven/aza sensitive LSCs and increased expression of genes involved in calcium efflux from the ER in ven/aza resistant samples. Ven/Aza resistant LSCs have increased mitochondrial calcium content, consistent with their increased OXPHOS activity as calcium is required for OXPHOS. Perturbation of these channels leads to decreased OXPHOS activity and decreased viability in LSCs.
DISCUSSION/SIGNIFICANCE OF IMPACT: We postulate that a deeper understanding of the mechanisms behind ven/aza targeting of LSCs will lead to the development of novel therapies for patients who do not respond to ven/aza. Our data show targeting intracellular calcium signaling could be a viable therapeutic strategy for AML patients.

The role of the L-type calcium channel, Cav1.3, in motor and associative learning

Aislinn Joanmarie Williams, University of lowa ${ }^{1}$, Marisol Lauffer, Hsiang Wen, and Bryn Myers

${ }^{1}$ University of lowa Institute for Clinical and Translational Science

OBJECTIVES/GOALS: Genetic variation in L-type voltage-gated calcium channels, including $\mathrm{Ca}_{\mathrm{V}} 1.3$, is associated with increased risk for psychiatric disorders including bipolar disorder and schizophrenia. Additionally, rare mutations in $\mathrm{Ca}_{\mathrm{V}} 1.3$ have been linked to epilepsy, developmental delay, and autism. Deletion of $\mathrm{Ca}_{\mathrm{V}} 1.3$ in mice is associated with impaired consolidation of contextual fear conditioning. Some studies have also observed affective behavior deficits in $\mathrm{Ca}_{\mathrm{V}}$ 1.3-deficient mice, but other studies have not found affective phenotypes, perhaps due to differences in genetic backgrounds, sex ratios, or task protocols. $\mathrm{Ca}_{\mathrm{V}} 1.3$ is important for slow afterhyperpolarization in hippocampal and amygdala neurons, which prevents excessive firing in response to sustained excitatory input, and $\mathrm{Ca}_{\mathrm{V}}$ 1.3-deficient amygdala neurons exhibit hyperexcitability and impaired LTP. $\mathrm{Ca}_{\mathrm{V}} 1.3$ is also expressed in the cerebellum, but its functional role there is not well understood. Given its importance in shaping neuronal activity in the hippocampus and amygdala, we hypothesized that loss of $\mathrm{Ca}_{\mathrm{V}} 1.3$ would cause abnormalities in motor learning as well as affective and cognitive behaviors. METHODS/STUDY POPULATION: Wild-type (WT), haploinsufficient (Hap), and knockout (KO) mice were maintained on a congenic $\mathrm{C} 57 \mathrm{BL} / 6 \mathrm{NTac}$ genetic background and were subjected to behavioral tasks including open field, rotarod, ErasmusLadder, elevated zero maze, forced swim test, and tail suspension test. Data were analyzed with sexes combined and with sexes separated to assess for sex as a biological variable. Studies were analyzed by one-way ANOVA, two-way ANOVA, or generalized linear mixed model, where appropriate. RESULTS/ANTICIPATED RESULTS: $\mathrm{Ca}_{\mathrm{V}} 1.3 \mathrm{KO}$ was associated with impaired motor learning in the rotarod task $(\mathrm{p}<0.05)$, as well as impaired associative learning in the ErasmusLadder task $(\mathrm{p}<0.01)$, despite intact locomotor function on both tasks. When examined by sex, the rotarod phenotypes were driven by motor learning impairments in males (both Hap and KO, $p<0.05$ and $p<0.01$, respectively), whereas the ErasmusLadder associative learning phenotypes were present in both sexes only in the $\mathrm{KO}$ condition, consistent with previously reported impairments in $\mathrm{Ca}_{\mathrm{V}}$ 1.3-deficient mice in consolidation of contextual fear conditioning. Although $\mathrm{KO}$ mice learned the motor aspects of the ErasmusLadder task, they learned more slowly. They also failed to learn start cues, which requires intact associative learning. No differences were observed in overall exploration or locomotor activity in open field or elevated zero maze. Analyses from affective tasks are ongoing. DISCUSSION/SIGNIFICANCE OF IMPACT: These preliminary studies provide new evidence that $\mathrm{Ca}_{\mathrm{V}} 1.3$ is important for the function of neural circuits involved in motor learning, and concur with previous data showing its involvement in associative learning. Our data differ slightly from previous studies of $\mathrm{Ca}_{\mathrm{V}} 1.3$ 